\title{
[Pyruvate Dehydrogenase [Lipoamide]] Kinase Isozyme 1, Mitochondrial
}

National Cancer Institute

\section{Source}

National Cancer Institute. [Pyruvate Dehydrogenase [Lipoamide]l Kinase Isozyme 1, Mitochondrial. NCI Thesaurus. Code C104761.

[Pyruvate dehydrogenase [lipoamide]] kinase isozyme 1, mitochondrial (436 aa, $49 \mathrm{kDa}$ ) is encoded by the human PDK1 gene. This protein is involved in pyruvate and glucose metabolism. 